\title{
CD31 density is a novel risk factor for patients with B-cell chronic lymphocytic leukaemia
}

\author{
TRYFONIA MAINOU-FOWLER ${ }^{1}$, ALISTAIR PORTEOUS ${ }^{1}$, ANNETTE NICOLLE $^{1}$, \\ STEPHEN JOHN PROCTOR ${ }^{1}$, JOHN JAMES ANDERSON ${ }^{1}$ and GEOFFREY SUMMERFIELD ${ }^{2}$
}

\author{
${ }^{1}$ Academic Haematology, School of Clinical and Laboratory Sciences, William Leech Building, The Medical School, \\ Framlington Place, Newcastle upon Tyne, NE2 4HH; ${ }^{2}$ Pathology, Queen Elizabeth Hospital, Gateshead, NE9 6SX, UK
}

Received February 20, 2008; Accepted March 24, 2008

\begin{abstract}
CD31 is the physiological ligand for CD38. CD38 expression in a high percentage of malignant cells is a risk factor for patients with B-cell chronic lymphocytic leukaemia (B-CLL). A previous investigation demonstrated that quantification of CD38 improves upon the prognostic value of the percentage expression. A recent study states that the percentage of CD31 expression is not predictive in B-CLL. We reassessed the predictive power of CD31 in a cohort of 120 patients with B-CLL. Peripheral blood cells were stained with PCP-labelled anti $(\alpha)$-CD19, FITC- $\alpha-C D 5$ and PE- $\alpha-$ CD31 antibodies. CD31 expression was quantified using beads of specific antibody binding capacity and the density was correlated with clinical outcome. End points were disease-specific survival and time to treatment (TTT). We report that $\mathrm{CD} 31$ density was significantly lower in the group of patients with Binet stage $\mathrm{B}$ and $\mathrm{C}$ of disease progression $(\mathrm{P}=0.0003)$. There was an inverse, significant correlation between CD31 and CD38 densities ( $\mathrm{R}=-0.281, \mathrm{P}=0.002)$. All CLL-related deaths occurred in patients with low CD31 density. Low CD31 predicted for poor disease outcome (survival, $\mathrm{P}=0.0087$; TTT, $\mathrm{P}=0.0064$ ) and identified Binet stage A patients (survival, $\mathrm{P}=0.0350$; TTT, $\mathrm{P}=0.0716$ ) and those with low $\mathrm{CD} 38$ (survival: all patients, $\mathrm{P}<0.0001$; stage $\mathrm{A}, \mathrm{P}=0.003$ ) who followed a more aggressive clinical course. Disease-specific survival of patients with low CD31 and high CD38 densities was significantly shorter than all other groups. In addition, low CD31 density was a poor risk factor irrespective of patient age (survival: all patients, $\mathrm{P}=0.045$; stage $\mathrm{A}, \mathrm{P}=0.021$ ) and identified patients with Binet stage $\mathrm{B} / \mathrm{C}$ as the highest risk group $(\mathrm{P}<0.0001)$.
\end{abstract}

Correspondence to: Dr Tryfonia Mainou-Fowler, Academic Haematology, School of Clinical and Laboratory Sciences, William Leech Building, The Medical School, Framlington Place, Newcastle upon Tyne, Tyne and Wear, NE2 4HH, UK

E-mail: tryfonia.mainou-fowler@ncl.ac.uk

Key words: CD31, CD38, B-cell chronic lymphocytic leukaemia, prognosis, antigen-quantification
In conclusion, low CD31 density is an adverse prognostic indicator in B-CLL. Also, low CD31 density enhances the prognostic power of CD38 density. The interaction between CD31 and CD38 and its clinical significance in B-CLL requires further investigation.

\section{Introduction}

A large number of prognostic factors are available to help predict outcome in patients with B-CLL $(1,2)$. Recently described, significant and independent prognostic factors include the mutation status of the immunoglobulin (Ig) heavy chain variable region (VH) (2), p53 mutations (2) and high expression of ZAP-70 $(3,4)$ and CD38 $(5)$.

CD38 acts as a receptor involved in the transduction of signals essential for cell activation and proliferation (6), survival (7) and apoptosis (8). Activation of these pathways is achieved after interaction of CD38 with its ligand which has been identified as the platelet endothelial cell adhesion molecule-1 or CD31 (9). The CD31 molecule, a member of the immunoglobulin (Ig) superfamily, is expressed on normal naive B-cells with unmutated $\mathrm{IgVH}$ genes and it is downregulated upon differentiation to memory cells (10). Crosslinking of CD31 may lead to either cell survival $(11,12)$ or apoptosis (13).

The CD38 (14) and CD31 (15) antigens are expressed in different types of normal vascular cells. Co-expression of these factors has been reported in malignant cells (16-19) and the CD38/CD31 phenotype has been implicated in the aggressiveness of some malignancies (16). In multiple myeloma (MM), CD38 and CD31 were expressed at high density in all patients while CD31 but not CD38 was absent in most patients with plasmablastic MM or plasma cell leukaemia (16). In patients with B-CLL, a higher ratio of CD38/CD31 in the lymph nodes compared with that of the peripheral blood (PB) or bone marrow (BM) correlates significantly with clinical parameters indicative of disease aggression including stage, lymphoma-like tumour distribution and a diffuse pattern of BM infiltration (19). By contrast, although CD38 and CD31 were co-expressed by PB B-CLL B-cells, no significant association between Rai stage and CD38/CD31 phenotype was reported (18). A study carried out by Ibrahim et al showed that the percentage of CD31 expression alone did not predict for disease outcome in 
B-CLL (17). However, a low percentage of co-expression of CD31 and CD38 identified a group of patients with a better prognosis than all the other groups (17).

Since the density of CD38 is a significantly better prognostic marker for patients with B-CLL than the percentage expression (20), we performed a retrospective study to reassess the predictive power of CD31 in B-CLL. We quantified CD31 expression using flow cytometry and beads of specific antibody binding capacity and correlated the density of CD31 with clinical outcome. We also examined the impact of CD31 expression on the predictive power of CD38, both factors expressed as density rather than as the percentage of cells expressing each antigen.

\section{Materials and methods}

Patient population. A cohort of 120 patients was studied. All patients were diagnosed as having B-CLL on the basis of clinical examination, peripheral blood count, absolute lymphocyte count, immunophenotyping and monoclonality of the $\kappa$ or $\lambda$ light immunoglobulin chain expression. The clinical outcomes for all patients were obtained from the patients' records. Patient selection was based on the availability of clinical histories, cryopreserved cells and laboratory findings, including CD38 density (20). Patient informed consent was obtained and ethical approval was granted by the Local Research Ethics Committee.

Cells. Peripheral blood mononuclear cells were isolated by centrifugation on Ficoll-Hypaque gradient (Nycomed, Sheldon, Birmingham, UK) and cryopreserved in tissue culture medium (RPMI-1640, Invitrogen Life Technologies, Paisley, UK) containing 10\% v/v dimethylsulphoxide (NBS Biologicals, Huntington, UK) and 20\% (v/v) heat-inactivated fetal calf serum (Invitrogen Life Technologies). Cells were stored at $-140^{\circ} \mathrm{C}$.

Expression of CD31 (percentage). Peripheral blood lymphocytes $\left(1 \times 10^{6}\right)$ were stained for 45 min with $10 \mu 1$ of peridinine chlorophyll protein-labelled anti $(\alpha)$-CD19 (Becton Dickinson, Cowley, Oxford, England), $10 \mu 1$ of fluorescein isothiocyanate-labelled $\alpha$-CD5 (Becton Dickinson) and $15 \mu \mathrm{l}$ of phycoerythrin-conjugated $\alpha$-CD31 (Pharmingen, supplied by Becton Dickinson) monoclonal antibodies, or with PE-labelled control of the same isotype (and amount) as the CD31 antibody (Ab) (IgG1, Pharmingen). Cell-fluorescence was measured on a FACScan flow cytometer as described elsewhere (20).

The period of cell storage at $-140^{\circ} \mathrm{C}$ did not affect CD31 expression. Dual samples from four patients were thawed at different time intervals and cells were stained for CD31 expression as described above. Student's paired t-test showed that the pairing was effective: correlation coefficient $(\mathrm{R})=0.9370 ; \mathrm{P}=0.1079$ (two-tailed).

Quantification of CD31 expression (density). The expression of CD31 was quantified by flow cytometry using microbeads of specific antibody binding capacity (ABC) (Quantum Simply Cellular kit; Sigma-Aldrich, Poole, Dorset, England) as described elsewhere (20). Briefly, $20 \mu 1$ of beads were labelled under the same conditions and with an equal amount of CD31 Ab as the experimental samples. Using incremental quantities of beads and the same amount of the test $\mathrm{Ab}$, it was revealed that the beads at $20 \mu 1$ were saturated with the Ab. A calibration curve was constructed as described elsewhere (20) and was used to obtain $\mathrm{ABC}$ values for the test samples. Storage at $-140^{\circ} \mathrm{C}$ did not affect $\mathrm{ABC}$ values; using dual samples thawed at different time intervals as described previously gave a $\mathrm{P}$-value of $0.3796 ; \mathrm{R}=0.6536$. The variation between the different calibration curves was insignificant ( $n=4$; mean of $R \pm S D, 0.9997 \pm 9.13 \times 10^{-5}$ ).

Statistical analyses. Fisher's exact and Spearman rank correlation tests were used to identify the cutoff point for disease outcome and the association between CD38 and CD31 expression, respectively. The Mann-Whitney U test was employed to examine any significant differences in the expression of CD31 between male and female patients or between patients with Binet stage A and stage B/C. KaplanMeier survival curves were plotted using SPSS for Windows, version 11 . P-values $<0.05$ were regarded as significant and between 0.05 and 0.1 as suggestive of a trend.

\section{Results}

Patient population. The cohort of 120 patients consisted of 70 males and 50 females, median age 70 years (range, 47-91; Table I). At diagnosis, 103 were in Binet stage A and 17 in stage B or C (Table I). Median follow up was 6.9 years (range: 7 months to 22 years 2 months). There were 32 deaths in the patient cohort, 22 from CLL (12 in stage A patients), four from ischaemic heart disease, two from sepsis and four from cancer. Fifty-seven patients had received treatment for CLL before entry to this study. The patient demographics are given in Table I.

CD31 density. All patients were positive for CD31. The mean percentage expression (SD) was $95.9 \%$ (2.87). The mean (SD) of the ABC values was: 30218 (13243). There was no correlation between CD31 density and age (Spearman's rank: $\mathrm{R}=0.02284, \mathrm{P}=0.8044$ ) and $\mathrm{CD} 31$ was equally distributed between the male and female groups (Mann-Whitney $\mathrm{U}$ test, $\mathrm{P}=0.3024)$. There was a significantly higher level of CD31 expression within the stage A group of patients than those in stage B and C (Fig. 1a, $\mathrm{P}=0.0003$, Mann-Whitney U test). The 75th percentile of CD31 density as $\mathrm{ABC}$ value (38294 ABC) was found to be the cutoff point that identified disease outcome (Fisher's exact). High density of CD31 (equal to or above the cutoff value of $38294 \mathrm{ABC}$ ) predicted for disease-specific survival (Fig. 2a; Kaplan-Meier, $\mathrm{P}=0.0087$ ) and time to requiring treatment (TTT) (Fig. 2f; Kaplan-Meier, $\mathrm{P}=0.0064$ ) and identified Binet stage A patients who had more aggressive disease (Figs. $2 \mathrm{~b}$ and $\mathrm{g}$; Kaplan-Meier: disease-specific survival, $\mathrm{P}=0.035$; TTT, $\mathrm{P}=0.0716)$. All CLL-related deaths occurred in patients with low CD31 (Table I). No patient with Binet stage B/C and high CD31 density was identified (Table I). Low CD31 density predicted for a more aggressive clinical course irrespective of patient age (Fig. $2 c, P=0.045$ and $2 d, P=0.021$ ) or stage of disease progression (Fig. $2 \mathrm{e}, \mathrm{P}<0.0001$ ). Patients 
Table I. Patient characteristics.

\begin{tabular}{lccccc}
\hline Patients & All patients & CD31 high & CD31 low & CD38 high & CD38 low \\
\hline Number & 120 & 30 & 90 & 27 & 93 \\
Male(M):Female(F) & $70: 50$ & $16: 14$ & $54: 36$ & $19: 8$ & $51: 42$ \\
Median age at diagnosis (range) & $70(47-91)$ & $73(52-91)$ & $69(47-90)$ & $68(47-90)$ & $71(49-91)$ \\
Binet A (M:F) & 103 & $30(16: 14)$ & $73(39: 34)$ & $14(8: 6)$ & $89(47: 42)$ \\
Binet B (M:F) & 8 & - & $8(6: 2)$ & $7(5: 2)$ & $1(1: 0)$ \\
Binet C (M:F) & 9 & - & $9(9: 0)$ & $6(6: 0)$ & $3(3: 0)$ \\
CLL deaths & 22 & - & 22 & 13 & 9 \\
\hline
\end{tabular}

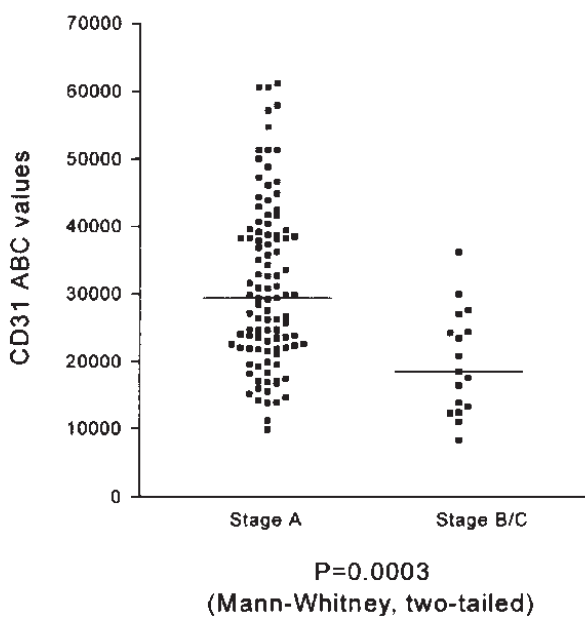

b

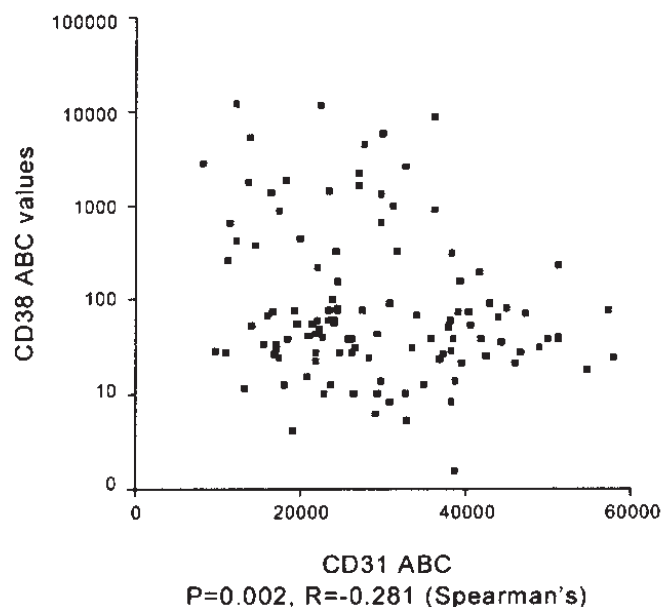

Figure 1. The density of CD31 expressed as ABC values was significantly higher in patients with Binet stage A disease (n=103) than in patients with progressive disease (Binet stage $\mathrm{B} / \mathrm{C} ; \mathrm{n}=17$ ) (a). The mean $\mathrm{ABC}$ values (SD) and confidence intervals (CI) were: stage A: $31141 \mathrm{ABC}(12345)$; $\mathrm{CI}$ : 33541 and 28740; and stage B/C: 20241 ABC (7804); CI, 24400 and 16082. There was a weak but significant inverse correlation between the densities of CD31 and CD38 (b).

with low $\mathrm{CD} 31$ and in stage $\mathrm{B} / \mathrm{C}$ or under 60 years of age had the worse prognosis (Figs. 2e, c and d, respectively).

CD31 and CD38 densities. When all patients were included in the analysis, there was a significant and inverse correlation between CD31 and CD38 densities (Fig. 1b).

To investigate the impact of CD31 density on the prognostic value of CD38 density, we used the CD38 ABC values of the same cohort of patients obtained in a previous study performed by our group (20). The cutoff point for CD38 density in this previous study was 250 ABC (20). Survival period of patients with low CD31 (below 38294 $\mathrm{ABC}$ ) and high CD38 (above or equal to $250 \mathrm{ABC}$ ) was significantly shorter than all the other groups (Fig. 3a and b, Kaplan-Meier). When all patients were included in the analysis, low CD31 identified those with low CD38 who had more aggressive disease (Fig. 3a, $\mathrm{P}=0.0425$ ). No patient with high density of both CD31 and CD38 was identified in the cohort of 120. There was a suggesting trend that low CD31 density predicted for disease outcome for Binet stage A patients with low CD38 (Fig. 3b, $\mathrm{P}=0.0721$ ).
Statistical analysis. In Cox multivariate analysis when all patients were included and stage, age, CD31 and CD38 densities were analysed, stage was the most significant predictor of survival $(\mathrm{P}<0.0001)$.

\section{Discussion}

This is the first report to show that the level of expression (density) on the malignant cells of CD31, the physiological ligand for $\mathrm{CD} 38$, is a significant prognostic indicator for patients with B-CLL.

Low CD31 density predicted for poor disease outcome for all patients and for Binet stage A patients separately. Also, low CD31 identified the group of patients with good prognosis (i.e. low CD38) who were at high risk of disease progression. Interestingly, all disease-related deaths occurred in patients with low CD31 while, regarding CD38 expression, there was almost an equal distribution of deaths between the group with low and that with high CD38 density. In addition, the mean value of CD31 density of the group of patients with progressive disease was significantly lower than the mean 

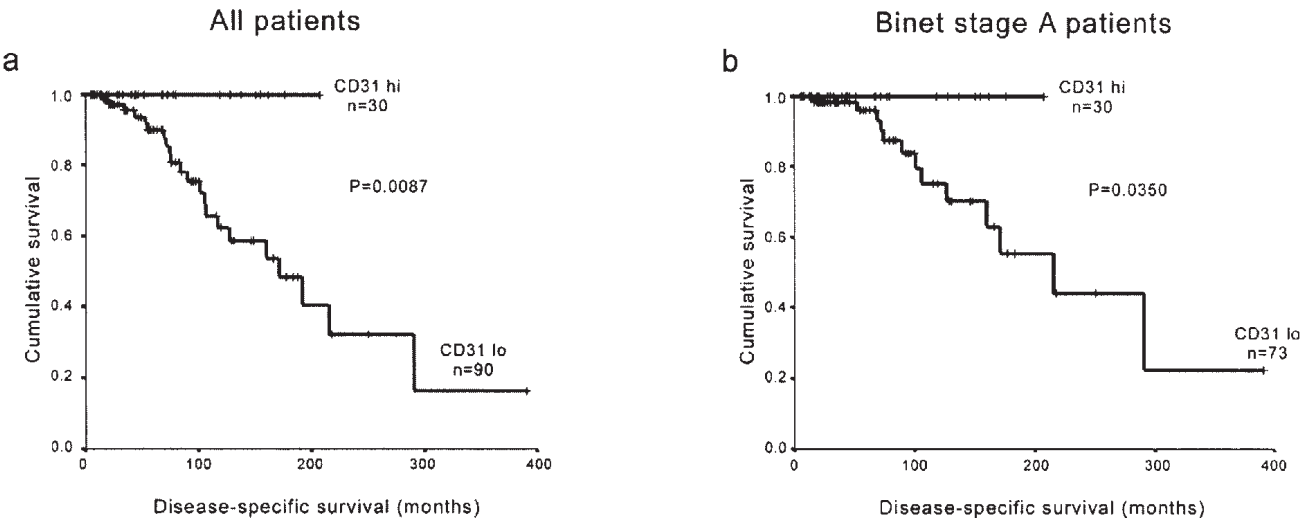

C
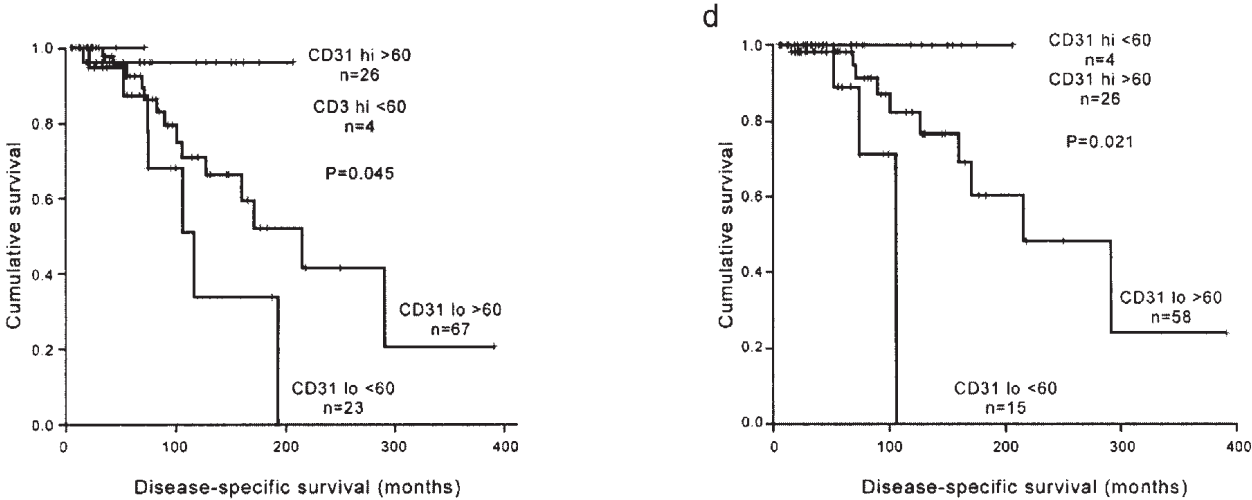

e

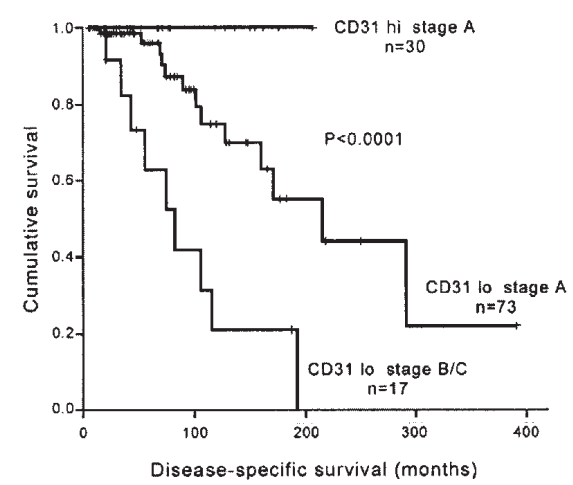

f
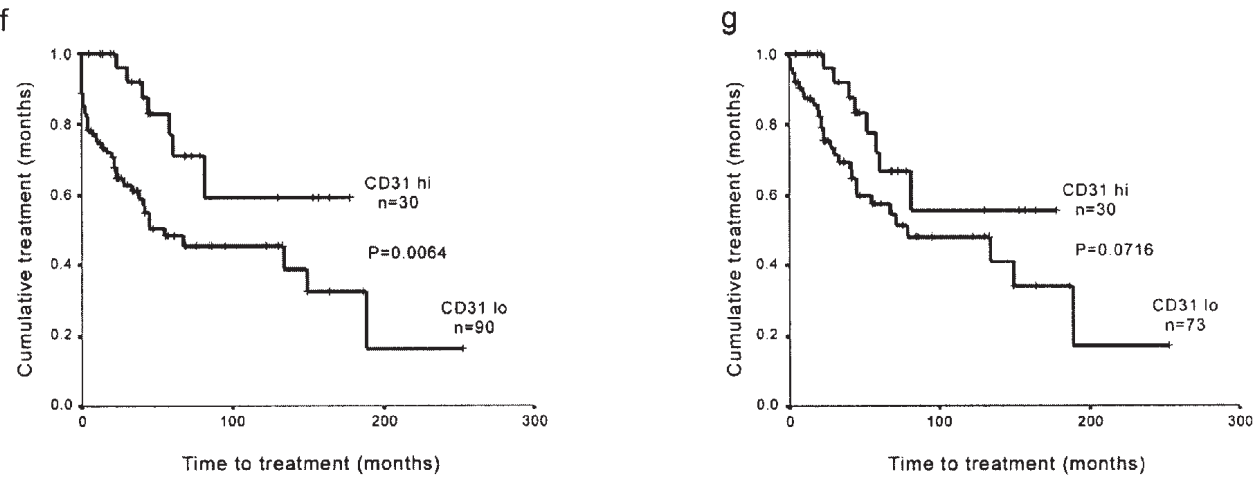

Figure 2. Disease-specific survival for all patients ( $a, c$ and e) and for Binet stage A patients (b and d) was plotted on the basis of high (hi) or low (lo) CD31 density taking the 75th percentile of expression (38294 ABC) as the cutoff point using Fisher's exact test. CD31 hi, $\geq 38294$ ABC; CD31 lo, <38294 ABC. (a and b) Disease-specific survival curves for 'all' and Binet stage A patients, respectively, based on CD31 hi or lo density. (c and d) Disease-specific survival of 'all' and Binet stage A patients, respectively, based on CD31 density and patient age. CD31 hi and >60 years vs CD31 lo and >60 years: 'all patients', $\mathrm{P}=0.082$; Binet stage $\mathrm{A}, \mathrm{P}=0.065$. CD31 lo and $>60$ years vs $\mathrm{CD} 31$ lo and $<60$ years: 'all patients', $\mathrm{P}=0.086$; Binet stage $\mathrm{A}, \mathrm{P}=0.050$. (e) Survival curve of patients on the basis of $\mathrm{CD} 31$ hi or lo and Binet stage $\mathrm{A}$ or B/C. CD31 hi Binet stage A vs CD31 lo Binet stage A, P=0.035; CD 31 lo Binet stage A vs CD31 lo Binet stage $B / C, P<0.0001$. CD31 hi Binet stage $A$ vs $C D 31$ lo Binet stage $B / C, P<0.0001$. (f and g) Kaplan-Meier curves representing time to treatment for all patients and for Binet stage A patients, respectively, based on high ( $\geq 38294$ ABC) or low (<38294 ABC) CD31 density. 


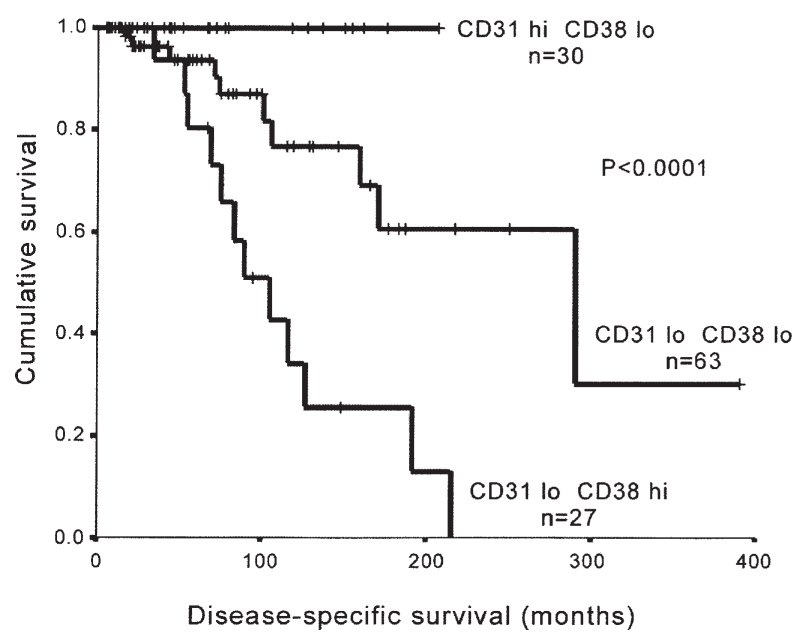

b

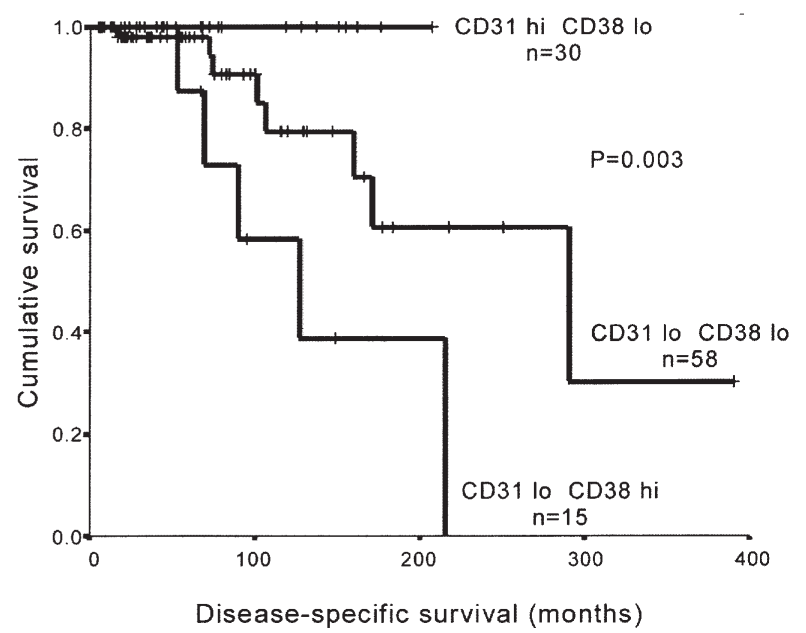

Figure 3. (a and b) Disease-specific survival of all patients and Binet stage A patients, respectively, on the basis of CD31 and CD38 densities. The cutoff points were: CD31 hi, $\geq 38294 \mathrm{ABC}$; CD31 lo, <38294 ABC and CD38 hi, $\geq 250 \mathrm{ABC}$; CD38 lo, $<250 \mathrm{ABC}$, as assessed in a previous study (20). CD31 hi CD38 lo vs CD31 lo CD38 lo: 'all patients', $\mathrm{P}=0.0425$; Binet stage $\mathrm{A}, \mathrm{P}=0.0721$. CD31 lo CD38 lo vs CD31 lo CD38 hi: 'all patients', $\mathrm{P}=0.0010$; Binet stage $\mathrm{A}, \mathrm{P}=0.0210$. $\mathrm{CD} 31$ hi CD38 lo vs CD31 lo CD38 hi: 'all patients', $\mathrm{P}<0.0001$; Binet stage $\mathrm{A}, \mathrm{P}=0.0030$.

value of the group in stage A disease. No patient with high $\mathrm{CD} 31$ and in Binet stage $\mathrm{B} / \mathrm{C}$ of disease progression was identified in this cohort of patients. These findings imply that low CD31 density may relate to disease overload and also that low CD31 is a better predictive indicator than high CD38 density. Furthermore, the presence of both adverse factors (i.e. high CD38 and low CD31) identified the group of patients with the highest risk of disease progression. Interestingly, no patient with high density of both factors was identified in this cohort. These findings prompt us to speculate that the level of expression of CD31 and its receptor $\mathrm{CD} 38$ by the malignant cells could partly govern disease aggression. Indeed, in patients with MM, CD38 and CD31 positivity (density) has been associated with the less aggressive forms of the disease while most patients with the more aggressive plasmablastic MM or plasma cell leukaemia express high levels of CD38 but CD31 is absent (16). Since CD31 is the counter-receptor for CD38 (9) and as both molecules are involved in the activation of cell survival $(7,11,12)$ or apoptosis pathways $(8,13)$, it is possible that these two molecules may interact with each other and the outcome of such interplay may affect disease progression by affecting the survival patterns of the malignant cells. Indeed, a study dating from 1996 reported that ligation of CD38 induced by its dimerization with a specific monoclonal antibody resulted in the activation of signal transduction pathways that inhibited proliferation and induced apoptosis of human immature B-cells (21). Since these activities were induced only upon CD38 ligation, these authors suggested the existence of a natural ligand for CD38 (21). In addition, a recent study showed that the ligation of CD31 with CD38 is involved in T-cell selection in the thymus and induces apoptosis of the double-positive thymocytes (22).

It is possible to hypothesise that in B-CLL, homophilic (with itself) or heterophilic (with CD38) interactions of CD31 (23) within the microenvironment of the lymph node
(LN) or BM may also lead to the activation of apoptotic pathways and the subsequent death of the leukaemic cells. Such interplay may act as a negative system to counteract the growth potential of the $\mathrm{CD} 38^{+}$malignant cells within the biological setting of BM and/or LN microenvironment where cytokines and interactions of the malignant cells with endothelial $\left(\mathrm{CD} 31^{+}\right)$and nurse-like cells enhance their proliferation and survival $(24,25)$.

In the present study, it is likely that the affinity of such interaction and, indirectly, the density of receptor and its counter-receptor, may be an important factor that governs the activation of apoptosis pathways. Such interaction between CD31 and CD38, both being expressed on malignant cells, may also provide an explanation for the poor disease outcome observed in some patients with B-CLL who express different levels of CD38, as it has been suggested (18).

In contrast to the findings of the present study, Ibrahim et al reported that CD31 expression alone did not predict for disease outcome but low co-expression of both CD31 and CD38 identified patients with a good prognosis (17). However, these authors examined the predictive value of the percentage of the co-expressed factors, while the present study investigated the prognostic significance of the densities of CD31 and CD38, both factors expressed on malignant cells. Furthermore, although CD31 and CD38 were co-expressed in patients with B-CLL, the CD38/CD31 phenotype did not associate significantly with the stage of disease (18). Again, this study examined the role of the percentage rather than the level of expression of CD31 and CD38 (18).

In conclusion, the density of CD31 identifies a group of patients with high expression who have a major survival advantage over patients with low expression. Low levels of CD31 identify patients with low CD38 who have a worse prognosis. The mode of action by which CD31 and CD38 affect the clinical outcome in B-CLL merits further investigation. 


\section{Acknowledgements}

The authors would like to thank Brian Shenton for helpful discussions regarding antigen quantification by flow cytometry and Lisa Thomas for assistance with flow cytometry. This study was supported by the Tyneside Leukaemia Research Association and Marrow and Stem Cell Transplant 2000.

\section{References}

1. Zwiebel JA and Cheson BD: Chronic lymphocytic leukemia: staging and prognostic factors. Semin Oncol 25: 42-59, 1998.

2. Stilgenbauer S, Bullinger L, Lichter P, Dohner $\mathrm{H}$ and The German CLL Study Group (GCLLSG): Genetics of chronic lymphocytic leukemia: genomic aberrations and $\mathrm{V}_{\mathrm{H}}$ gene mutation status in pathogenesis and clinical course. Leukemia 16: 993-1007, 2002.

3. Del Principe MI, Del Poeta G, Buccisano F, et al: Clinical significance of ZAP-70 protein expression in B-cell chronic lymphocytic leukemia. Blood 108: 853-861, 2006.

4. Orchard JA, Ibbotson RE, Davis Z, et al: ZAP-70 expression and prognosis in chronic lymphocytic leukemia. Lancet 363: 105111,2004

5. Mainou-Fowler T, Dignum HM, Proctor SJ and Summerfield GP: The prognostic value of CD38 expression and its quantification in B cell chronic lymphocytic leukemia (B-CLL). Leuk Lymphoma 45: 455-462, 2004.

6. Funaro A, Spagnoli GC, Ausiello CM, et al: Involvement of the multilineage CD38 molecule in a unique pathway of cell activation and proliferation. J Immunol 145: 2390-2396, 1990.

7. Zapo S, Rugari E, Dono M, Taborelli G, Malavasi F and Ferrarini M: CD38 signaling by agonistic monoclonal antibodies prevents apoptosis of human germinal center B cells. Eur J Immunol 24: 1218-1222, 1994.

8. Kumagai M, Coustan-Smith E, Murray DJ, et al: Ligation of CD38 suppresses human B lymphopoiesis. J Exp Med 181: 1101-1110, 1995

9. Deaglio S, Morra M, Mallone R, et al: Human CD38 (ADPribosyl cyclase) is a counter-receptor of CD31, an Ig superfamily member. J Immunol 160: 395-402, 1998.

10. Jackson DE, Gully LM, Henshall TL, Mardell CE and Macardle PJ: Platelet endothelial cell adhesion molecule-1 (PECAM-1/CD31) is associated with a naïve B-cell phenotype in human tonsils. Tissue Antigens 56: 105-116, 2000.

11. Zocchi T and Poggi A: PECAM-1, apoptosis and CD34 ${ }^{+}$ precursors. Leuk Lymphoma 45: 2205-2213, 2004.
12. Gao C, Sun W, Christophidou-Solomidou M, et al: PECAM-1 functions as a specific and potent inhibitor of mitochondrialdependent apoptosis. Blood 102: 169-179, 2003.

13. Ilan N, Mohsenin A, Cheung L and Madri JA: PECAM-1 shedding during apoptosis generates a membrane-anchored truncated molecule with unique signalling characteristics. FASEB J 15: 362-372, 2001

14. Deaglio S, Metha K and Malavasi F: Human CD38: a (r)evolutionary story of enzymes and receptors. Leuk Res 25: 1-12, 2001

15. Newman PJ: Switched at birth: a new family for PECAM-1. J Clin Invest 103: 5-9, 1999

16. Vallario A, Chilosi M, Adami F, et al: Human myeloma cells express the CD38 ligand CD31. Br J Haematol 105: 441-444, 1999

17. Ibrahim S, Jilani I, O'Brien S, et al: Clinical relevance of the expression of CD31 ligand for CD38 in patients with B-cell chronic lymphocytic leukaemia. Cancer 97: 1914-1919, 2003.

18. Morabito F, Mangiola M, Stelitano C, Diaglio S, Callea V and Malavasi F: Simultaneous expression of CD38 and its ligand CD31 by chronic lymphocytic leukaemia B-cells: anecdotal observations or pathogenetic hypothesis for the clinical outcome? Haematologica 88: 354-355, 2003.

19. Jaksic O, Paro MMK, Skelin IK, Kusec R, Pejsa V and Jaksic B CD38 on B-cell chronic lymphocytic leukemia cells has higher expression in lymph nodes than in peripheral blood or bone marrow. Blood 103: 1968-1969, 2004.

20. Mainou-Fowler T, Dignum H, Taylor PRA and Dickinson AM Quantification improves the prognostic value of CD38 expression in B-cell chronic lymphocytic leukaemia. Br J Haematol 118: 755-761, 2002.

21. Silvennoinen O, Nishigaki H, Kitanaka A, et al: CD38 signal transduction in human B cell precursors. Rapid induction of tyrosine phosphorylation, activation of syk tyrosine kinase, and phosphorylation of phospholipase C-gamma and phosphatidylinositol 3-kinase. J Immunol 156: 100-107, 1996.

22. Tenca C, Merlo A, Zarcone D, et al: Death of T cell precursors in the human thymus; a role for CD38. Int Immunol 15: 11051116, 2003.

23. Jackson DE: The unfolding tale of PECAM-1. FEBS Lett 540: 7-14, 2003

24. Deaglio S, Capobianco A, Bergui L, et al: CD38 is a signaling molecule in B-cell chronic lymphocytic leukemia cells. Blood 102: 2146-2155, 2003.

25. Deaglio S, Vaisitti T, Aydin S, Ferrero E and Malavasi F: Intandem insight from basic science combined with clinical research: $\mathrm{CD} 38$ as both marker and key component of the pathogenetic network underlying chronic lymphocytic leukemia. Blood 108: 1135-1144, 2006. 